\title{
Engineering a Clinical Force Measuring Walker for Patients Recovering from Median Sternotomy
}

\author{
Ansel K. LaPier \\ Physical Therapy, Eastern Washington University, Spokane, WA 99202, USA \\ *Corresponding author: Ansel K. LaPier; LaPierLabHSB233@gmail.com
}

Received 11 December 2021;

Accepted 26 December 2021;

Published 01 January 2022

\begin{abstract}
Background: Patients often need the use of their arms to assist with functional activities, but after median sternotomy, pushing is frequently limited to less than $4.5 \mathrm{~kg}$. No method exists to objectively measure arm weight bearing in clinical settings. This project aimed to design, construct, and test a walker for patients recovering from median sternotomy to prevent excessive bone stress during ossification. Methods: First, a qualitative study was conducted to obtain critiques of a Clinical Force Measuring (CFM) Walker prototype from rehabilitation professionals. Key statements and phrases were coded that allowed "themes" to emerge from transcribed interviews, which guided device revisions. Next, a second CFM Walker prototype was designed based on the qualitative data and device criteria/constraints and finally tested. $\underline{\text { Results: }}$ The result was fabrication of a new lightweight, streamlined, and cost-effective prototype walker with a simple visual display and auditory cue with upper limit alarms. Key features included attachments for medical equipment and thin film force-sensing resistors integrated into the walker handles that progressively activated 3 LEDs and a buzzer when arm force exceeded programmed thresholds. Conclusions: The innovative CFM Walker will help patients with restricted upper extremity weight bearing, especially elderly adults, recover safer and faster in the future.
\end{abstract}

Keywords: Median sternotomy, feedback training, sternal precautions, instrumented walker, cardiac rehabilitation

\section{Introduction}

Patients recovering from bone disruption due to trauma or surgery need to limit use of their arms during bone healing often to less than $4.5 \mathrm{~kg}$ of pushing, pulling, or lifting ${ }^{[1-3]}$. This restriction is thought to minimize shear force and movement between the bone halves to protect callus formation and osteogenesis ${ }^{[1-5]}$. Median sternotomy is commonly performed to access the heart during a variety of different surgeries such as coronary artery bypass, heart valve replacement, heart transplantation, and thoracic trauma repairs. The procedure entails sawing longitudinally from the sternal notch to the xiphoid process, separating the sternum with retractors, and wiring the sternal halves together after surgery completion ${ }^{[6,7]}$. Complications can occur when the bone halves do not heal correctly, including deep wound infection (osteomyelitis), bony non-union/instability, and or bone dehiscence ${ }^{[1-4]}$. But, restricting arm use often limits patients' functional independence, contributing to longer hospital stays and greater need for care after hospitalization. It is difficult to function independently when upper body daily activities are limited, especially for older adults. Restricting arm use is particularly problematic for patients who need assistance sitting down or standing up from a chair or need to use a walker for ambulation. Loss of functional independence can contribute to a greater need for assistance and rehabilitation after hospital discharge ${ }^{[8-11]}$. Therefore, appropriate arm use is essential for timely return to function.

Little is known about how much upper extremity weight bearing (UEWB) force occurs when patients attempt to use less than $4.5 \mathrm{~kg}$; therefore, their ability to safely resume activity and use of a walker is unknown ${ }^{[12-14]}$. Adams et al. found that force when using a single arm to assist with standing up from a bench was $27.5 \mathrm{lb}(12.5 \mathrm{~kg}){ }^{[15]}$. Similarly, Swanson et al. found UEWB while moving from side lying to sitting in a bed was $22.2 \mathrm{lb}(10.1$ $\mathrm{kg}{ }^{[16]}$. Previously, we have shown that self-selected arm force when instructed to use less than $4.5 \mathrm{~kg}$ was $5.3-8.6 \mathrm{~kg}$ during ambulation with an assistive device and sit-stand transfers in a young (18-40 years old) cohort and that subjects used more than $5.5 \mathrm{~kg}$ of arm force during most trials $(67 \%)^{[12]}$. This study also employed a feedback training protocol and demonstrated its efficacy for improving subjects' ability to modulate UEWB. We have also corroborated these findings in a cohort of older subjects (60-85 years) and identified metrics predictive of excessive UEWB during functional tasks, including handgrip strength, static and dynamic balance, health status, and body mass index ${ }^{[13,14]}$. Other researchers have found that patients are not good at limiting leg weight bearing and can improve accuracy with feedback training [17-19].

Therefore, a method to objectively measure UEWB while patients use a walker is needed. Currently, there are no walkers for 
use with patients to provide UEWB feedback. The device I previously fabricated using handgrip dynamometers to measure UEWB was only appropriate for research purposes because it was bulky, had remote force displays, and was expensive. Other researchers have used bathroom scales, force plates/pressure sensing mats, or foot pressure sensors to measure extremity weight bearing ${ }^{[17-21]}$. Existing walkers instrumented to measure UEWB are only appropriate for research applications and have limitations that preclude their use with patients in clinical settings, including they: 1) have complicated force displays positioned remotely from the walker ${ }^{[12-14,22]}, 2$ ) use sensors placed in the walker's legs, not the handles $\left.{ }^{[22-24]}, 3\right)$ do not display data for the patient and therefore cannot be used for feedback training ${ }^{[24,25]}$, and 4) are bulky, expensive, and not built on a clinical walker frame ${ }^{[26]}$.

The ultimate goal or this project was to design and construct an instrumented walker for rehabilitation professionals to utilize with patients who need to restrict UEWB to safely perform functional mobility tasks using less than $4.5 \mathrm{~kg}$ of force. We know from previous work that UEWB and pectoralis major muscle electromyography during functional mobility are greater in younger versus older subjects and improve (decrease) following feedback training ${ }^{[12-14]}$. This preliminary research established proof-of-concept, the need for an instrumented walker, and the efficacy of its use with feedback training. The purpose of the first part of this project (Part 1) was to systematically obtain qualitative critiques from hospital rehabilitation professionals regarding an initial Clinical Force Measuring (CFM) walker prototype (v1.0) to guide revisions and refinement of the mechanical device and user interface. The engineering goal for the second part (Part 2) of this project was to use the information obtained in Part 1 to design and construct a second CFM Walker prototype (v2.0) and to test its ability to meet essential criteria and constraints.

\section{Materials and Methods}

\section{Qualitative Research Study (Part 1)}

First, a prototype was fabricated using biomedical device engineering and critical care equipment principles ${ }^{[27,28]}$. The CFM Walker v1.0 (see Figure 1) retained the externally mounted force transducers wirelessly connected to tablets. The tablets were housed in waterproof cases that could be disinfected and mounted directly to the walker with multi-planar adjustable mounting arms. A plate was attached below the left lateral support to suspend a chest tube reservoir tank with placement below the tube exit site to maintain gravity assist drainage of pleural secretions. Chest tubes exit the left lower chest wall after cardiac surgery and must have a water seal to maintain negative pressure within the pleural space. Walker legs were color-coded to facilitate adjustment for multiple patient use and included interchangeable front standard and 12.7 $\mathrm{cm}$ single plane wheeled legs. A portable oxygen tank mounting bracket was positioned on the lower front horizontal walker support and centred for optimal walker stability and symmetrical drag when using a front wheeled walker configuration. Ergonomic soft handle grips were added to improve patient ability to hold and patient comfort. A hook that rotated 90 degrees to suspend a urinary collection bag was mounted below the right lower lateral support to facilitate gravity assist drainage. The swivel hook allowed a urinary collection bag with a parallel or perpendicular oriented hook to hang parallel from the walker to keep it from obstructing gait. In addition, an S-shaped hook was attached directly to the right lower lateral support to tether a Foley Catheter (aka urinary catheter) in front of the patient's leg, so the tubing would not obstruct gait.

This part of the study used a qualitative description methodology (phenomenology). Qualitative inquiry is appropriate when seeking to describe a topic in depth through insights from participants ${ }^{[29,30]}$. By using an interview process, participants' perspectives were explored using open and probing questions. A purposeful sampling strategy was employed to achieve sufficient variability and understanding of the concepts. Study participants were rehabilitation professionals with experience working in critical care with post-surgical patients. Criteria for selection of subjects included: 1) between the ages of 25-60 years, 2) rehabilitation professional (physical therapist, registered nurse, exercise physiologist, etc.), and 3) minimum of 6 months working in a hospital with critical care patients. This study was approved by the Eastern Washington University Institutional Review Board for Human Subjects (Protocol \#HS-5953), and all subjects signed an informed consent prior to participation; procedures were in accordance with the Helsinki Declaration.
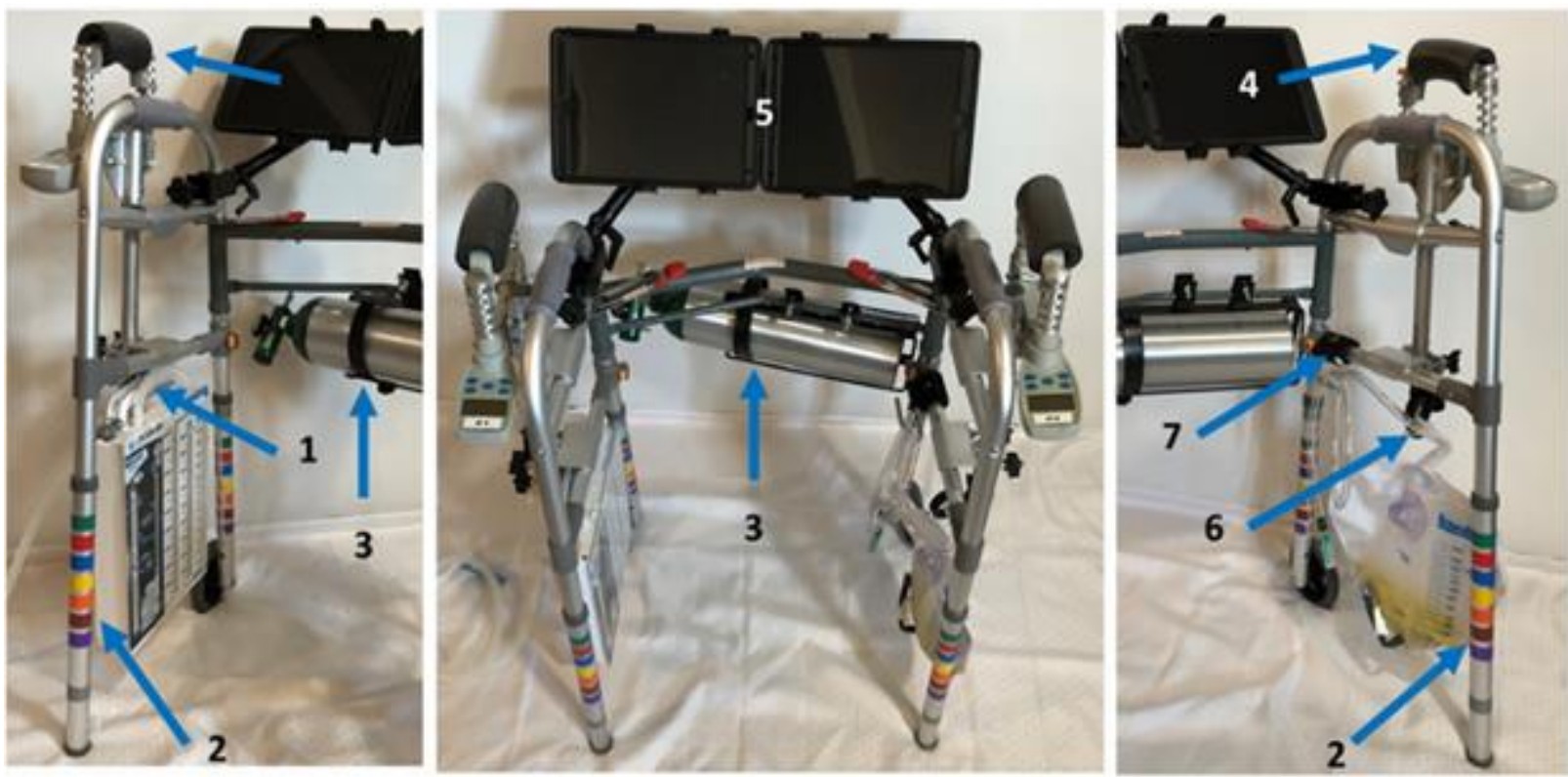

Figure 1: Clinical Force Measuring Walker v1.0 components: 1) Chest tube reservoir plate, 2) Color coded walker legs, 3) Oxygen tank mounting bracket, 4) Ergonomic soft handle grips, 5) Tablet adjustable mounting arms \& waterproof cases, 6) Urinary collection bag swivel hook, 7) Foley Catheter S-hook. 
Data collection involved asking the study participants a number of open-ended questions to garner feedback on the instrumented walker prototype. Subject interviews were conducted via Zoom virtual meeting application to adhere with COVID-19 social distancing guidelines. The interviews were video-recorded so that the answers and comments could be transcribed for data analysis. The subject interviews began with an introduction and narrated video clips of the walker prototype from multiple angles. The interview questions were sequentially shown on slides with close-up photographs of the specific walker parts/features from different angles.

Data analysis involved reviewing the transcribed interviews and identifying key statements and phrases that were significant within each category. Statements then were sorted into groups of similar ideas that emerged as meaningful units or "themes." Rich descriptions of participant perceptions corresponding with each theme were generated using their exact words and phrases. An iterative process of data analysis was used until saturation was achieved within each theme. Qualitative data were analysed and triangulated before themes were named.

\section{Engineering Design and Testing (Part 2)}

Figure 2 shows the flow of steps used to design the final CFM walker prototype. Using data from the qualitative study and device testing, extensive revisions were made to the first walker prototype. The essential features that the CFM Walker needed to have were defined based on engineering design criteria and constraints, qualitative data, and published information ${ }^{[27,28]}$. Next, testing procedures for each of the essential design elements were developed. Table 1 outlines the design elements, criteria, constraints, and protocols used to test both CFM Walker prototypes.

\section{Results and Discussion}

\section{Qualitative Research Study (Part 1)}

The total cost for the components to fabricate the CFM Walker v1.0 (Figure 1) was $\$ 1,423$. The bulk of this cost was for the force transducers and tablet displays. The total cost did not include the actual medical devices (portable oxygen tank, chest tube, chest tube reservoir, urinary collection bag, and Foley / urinary catheter).

Table 2 outlines the main themes for each walker component that emerged with data analysis. There were 5 overarching ideas that developed at the completion of data analysis.

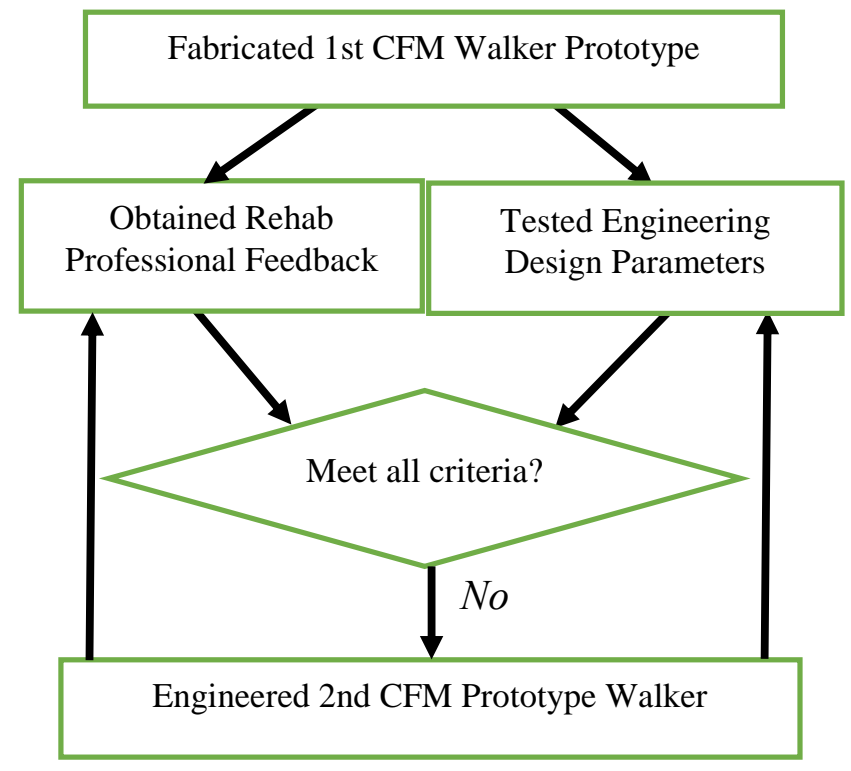

Figure 2: Engineering process flowchart.

$\mathrm{CFM}=$ Clinical Force Measuring

Table 1: Engineering Design Elements, Criteria, Constraints, and Testing Plan.

\begin{tabular}{|c|c|c|}
\hline Design Elements & Criteria / Constraints & Design Testing Plan *3 Trials \\
\hline $\begin{array}{l}\text { Vertical force measuring } \\
\text { capability }\end{array}$ & $\begin{array}{l}\text { Measurement accuracy } \geq 90 \% \text { in } 0.5- \\
9.1 \mathrm{~kg} \text { range }\end{array}$ & $\begin{array}{l}\text { Measurement using push dynamometer }{ }^{1} \text { on handle within correct } \\
\text { range: Green }<7 \mathrm{lb}(3.2 \mathrm{~kg}) \text {, yellow } 7-10 \mathrm{lb}(3.2-4.5 \mathrm{~kg}) \text {, red } 10 \mathrm{lb}(> \\
4.5 \mathrm{~kg})(30 \text { trials })\end{array}$ \\
\hline Ergonomic handles & Handle diameter $3-6 \mathrm{~cm}$ & $\begin{array}{l}\text { *Measure circumference of handles using tape with } 1 \mathrm{~mm} \text { increments } \\
\text { and calculate diameter }\end{array}$ \\
\hline $\begin{array}{l}\text { Simple visual \& auditory } \\
\text { feedback with alarms }\end{array}$ & $\begin{array}{l}\text { Display readable and buzzer audible } \\
\text { from } 1 \mathrm{~m} \text { with upper limit alarm }\end{array}$ & $\begin{array}{l}\text { *Measure distance in } 50 \mathrm{~cm} \text { increments up to } 3 \mathrm{~m} \text { that subjects ages } \\
18-83 \text { year old }(\mathrm{n}=6) \text { can: } 1) \text { see visual display screen and } 2) \text { hear } \\
\text { auditory signal output. }\end{array}$ \\
\hline $\begin{array}{l}\text { Streamlined, stable, } \& \\
\text { maneuverable frame }\end{array}$ & $\begin{array}{l}\text { Width }<66 \mathrm{~cm} \\
\text { Depth }<63 \mathrm{~cm}\end{array}$ & $\begin{array}{l}\text { *Measure using a caliper device and tape measure with } 1 \mathrm{~mm} \\
\text { increments }\end{array}$ \\
\hline Lightweight construction & Total weight $<6 \mathrm{~kg}$ & *Weigh walker with \& without attachments using scientific scale ${ }^{2}$ \\
\hline Minimal drag & $\begin{array}{l}\text { Horizontal push-pull resistance }<2 \\
\mathrm{~kg} \text { on solid surface }\end{array}$ & $\begin{array}{l}\text { Measure horizontal resistance using a push-pull force dynamometer } \\
\text { over } 155 \mathrm{~cm} \text { with } 4 \text { front wheel types }{ }^{3}(10 \text { trials each) }\end{array}$ \\
\hline Adjustable height handles & Appropriate for patients $1.6-1.8 \mathrm{~m}$ tall & $\begin{array}{l}\text { *Measure top of handle height using a caliper device and tape } \\
\text { measure with } 1 \mathrm{~mm} \text { increments }\end{array}$ \\
\hline Ability to disinfect & $\begin{array}{l}\text { All components nonporous; Electrical } \\
\text { components covered / water resistant }\end{array}$ & $\begin{array}{l}\text { Create checklist of component materials to categorize as nonporous vs } \\
\text { porous; Assess functionality after spraying } 100 \mathrm{cc} \text { of water }\end{array}$ \\
\hline Affordable cost & $\begin{array}{l}\text { Total cost of all components (parts } \\
\text { and materials) }<\$ 500\end{array}$ & Keep a detailed itemized list of all components and material costs \\
\hline
\end{tabular}

${ }^{l}$ Mark-10 CG High capacity digital force gauge, $453.6 \mathrm{~kg}$ tensile or compressive force (Mark-10 Corporation, Copiague, $\mathrm{NY}$ ); ${ }^{2} \mathrm{CAS} \mathrm{SW}-50 \mathrm{SW}$ $1 W$ Series Washdown Portion Control Bench Scale, $23 \mathrm{~kg}$ Capacity, $0.05 \mathrm{~kg}$ Readability (CAS Corporation, East Rutherford, NJ); ${ }^{3}$ Walker Wheels Swivel $12.7 \mathrm{~cm}$, Universal $7.6 \mathrm{~cm}$ (Drive Medical, Post Washington, NY), Lumex Swivel $7.6 \mathrm{~cm}$ (Graham Field, Atlanta, GA)

1. The subjects (rehabilitation professionals) overwhelmingly expressed that a force measuring walker would be very useful with a variety of patients, particularly those recovering from open heart surgery. 
2. Integrating the force measuring mechanism into the walker handles / structure would be optimal for arm biomechanics and the width of the device (to be manoeuvrable in narrow spaces).

3. Simplifying the force output display and adding upper limit visual and auditory alarms would be easier for patients and rehabilitation professionals to know when UEWB exceeds $4.5 \mathrm{~kg}$.

4. The optimal leg combination was unanimously front 12.7 $\mathrm{cm}$ single plane wheels and back standard legs.

5. Subjects also identified revisions to improve the medical equipment attachments for the oxygen tank, Foley Catheter, and oximeter.

Table 2: Qualitative Data Themes for Each Walker Component.

\begin{tabular}{l} 
Clinical Force Measuring Walker v1.0 Themes \\
\hline Chest Tube \& Reservoir \\
- Good location - low for drainage, same location often used clinically \\
- Tube adequately protected (not touching ground, kinked, tangled) \\
- Essential features: no gait obstruction, protected adequately, user-friendly \\
- May not work for all reservoir types (with different shapes, handles, hooks) \\
- Improvements: higher or with adjustable height, block swinging inward
\end{tabular}

\section{Walker Legs}

- Ideal combination: wheels only on front legs, commonly used clinically

- Color-coding possibly helpful, not necessary (Healthcare professionals already familiar with this type of height adjustment mechanism)

- Improvements: color-coding material needs to be nonporous for disinfecting, back leg "ski-type" gliders

\section{Oxygen Tank}

- Not a good location - tendency to tip forward, cause asymmetrical drag

- Essential features: no gait obstruction, protected adequately, user-friendly

- Would not fit most common portable oxygen tanks used in hospitals

- Improvements: remove bracket, transport tank separately / with other device (IV pole), better to have another person to assist with oxygen tank if needed

\section{Force Transducers}

- Wider diameter grips better for patients

- Transducer handles wider than normal - problematic for small patients, hard to fit through narrow spaces, change biomechanics of arm force

- 2 sets of hand grips confusing for patients

- Improvements: materials need to be nonporous for disinfecting, integrated transducers ideal to reduce width / weight and simplify build

\section{Display Screen Mounts}

- Location Issues: possibly cause tipping forward, obstruct patient view while walking, hard for provider to see if patient is large

- Essential features: good adjustability with multi-angle articulation, wireless connection, intuitive, easy to use

- Improvements: single unit instead of 2, reduce weight and size

\section{Display Interface}

- Good to have units in pounds for patient reference

- Color-coded, graphical information helpful

- Visual feedback display too complicated and small

- Improvements: larger, simpler force output, upper limit signal warning lights (flashing lights, color LEDs) and auditory signal (buzzer)

\section{Urinary Collection Catheter \& Bag}

- Good location - low for drainage, same location often used clinically

- Catheter adequately protected (not touching ground, kinked, tangled)

- Essential features: no gait obstruction, protected adequately, user-friendly

- Improvements- block swinging inward, remove hook for catheter

\section{Overall Opinion}

- Force measuring walker with integrated handles clinically useful

- Streamline attachments to reduce total weight and surface area

- $\quad$ Some attachments helpful; remove oxygen tank, add oximeter

- Simplify visual display and add auditory warning signal

- Useful for a variety of patient populations (median sternotomy, arm fracture / surgery, critically ill...)

Direct quotes were extracted from the transcribed data to provide rich descriptions of the subjects' perceptions corresponding with each theme. A few example quotes for each walker component are provided here.

\section{Chest Tube \& Reservoir}

- I think it would protect it better than I do when I walk with them [hung on side walker]. 
- I like that the location is low so it shouldn't impede drainage.

\section{Walker Legs}

- I actually prefer that size of wheel [12.7 $\mathrm{cm}$ single plane] vs the smaller circumference...swivel wheels just add another plane of movement [so not ideal].

- We are familiar with the button [mechanism to change height]. So, you don't need to [change it].

\section{Oxygen Tank}

- I don't know that you necessarily need the oxygen tank attached.

- ...it's attached to other equipment like IV poles that I'm already taking with me.

\section{Force Transducers}

- It might be confusing for patients who are reaching up to grab the walker to have 2 sets of handles.

- So as it is right now, it might be difficult to fit say in and out of the bathroom doorway...

\section{Display Screen Mounts}

- $\quad$...would be in the way as far as patients being able to see where they're going or looking for obstacles.

- [I] like that they have a lot of degrees of freedom.

\section{Display Interface}

- ...having the screen flash if you went over the set amount would be really beneficial for that feedback.

- [Good] to have an annoying buzzer just when they've gone over their mark- because they're pretty good at hearing annoying things.

\section{Urinary Collection Catheter and Bag}

- I think it would protect it better than I do when I walk with them.

- I like the hook because it can't slide.

- Looks fabulous!

\section{Overall Opinion}

- I would love to see the next generation of- when you upgrade this walker. This is already innovative. Very practical, but it's just a bit busy. -this is the right way to go forward. Advancing in terms of how we improve our patients' gait.

- I like the ability to measure force that the patients are using in their arms...I've never seen that before... and to have some built-in attachments.

- ...it's beneficial across a broad range of patients.
- $\quad$...you could tell how much of their body weight they were putting through their legs versus their arms. So, you know how much they were relying on the walker to assist with ambulation.

\section{Engineering Design and Testing (Part 2)}

The component locations of the CFM Walker v2.0 are shown in Figure 3. Thin-film force resistors $(1.8 \times 1.8 \mathrm{~cm})$ were placed under the original walker handgrips (Figure 4.A). Khodadadi et al found that force transducers incorporated into walker handles had easier installation and less error than those in-stalled on circular vertical walker legs ${ }^{[25]}$. These were connected to the Arduino System with male-to-female breadboard jumper wires. A visual display with 3 different coloured LEDs was designed to simplify the force feedback interface. The LEDs were triggered as follows: the green LED was always on, the yellow LED was activated when force was greater than or equal to $7 \mathrm{lb}(3.2 \mathrm{~kg})$, and the red LED was activated when force was greater than or equal to $10 \mathrm{lb}(4.5$ $\mathrm{kg}$ ). An auditory alarm that triggered when force exceeds $4.5 \mathrm{~kg}$ was also included. The system was programmed to trigger the red LED and buzzer when the left or right force transducer measured greater than the pre-set $4.5 \mathrm{~kg}$ of force. The thin-film force resistors were calibrated with a force dynamometer in a 1-20 lb (0.5-9.1) kg range. Force accuracy data are presented in Figure 5. An external power source was added to the system, and Figure 4.C illustrates the electrical schematic of the feedback system. The electrical components were housed in a clear acrylic waterproof case with exit holes for the 3 LEDs, speaker, and cord to the power source (Figure 4.E). The electrical component housing and external power source were positioned on the front of the walker using a multi-planar clamp mount. This position allowed the patient and healthcare professional to see the LEDs but did not obstruct their view when walking forward.

A bracket fabricated to hold a standard handheld oximeter and was clamped to the right upper vertical walker support. This position allowed healthcare providers to maintain line-of-sight for continuous oxygen saturation and heart rate monitoring. In this position, the oximeter probe remained attached to the patient's finger while using the walker. The oximeter holder could also be placed on the left side or other horizontal or vertical walker support. The bracket was designed to make the digital display visible and allow easy oximeter placement and access to the power switch (Figure 4.D). A swivel hook was mounted to the upper right horizontal support using a metal hose clamp to suspend a urinary collection bag (Figure 4.B). A 90 degree swivel hook was used so urinary collection bags with a parallel or perpendicular suspension hook could be attached to the walker and maintain bag orientation in the same plane as the right walker support frame. By moving this swivel hook to a higher location, the S-hook to tether the Foley Catheter was no longer needed. This higher attachment location ensured that the urinary collection bag would not drag on the floor; it also could not swing inward, hitting the patient's foot because the lower right horizontal support blocked this motion. Even with this higher location, the urinary collection bag was still lower than the level of the bladder to maintain gravity assist drainage. 

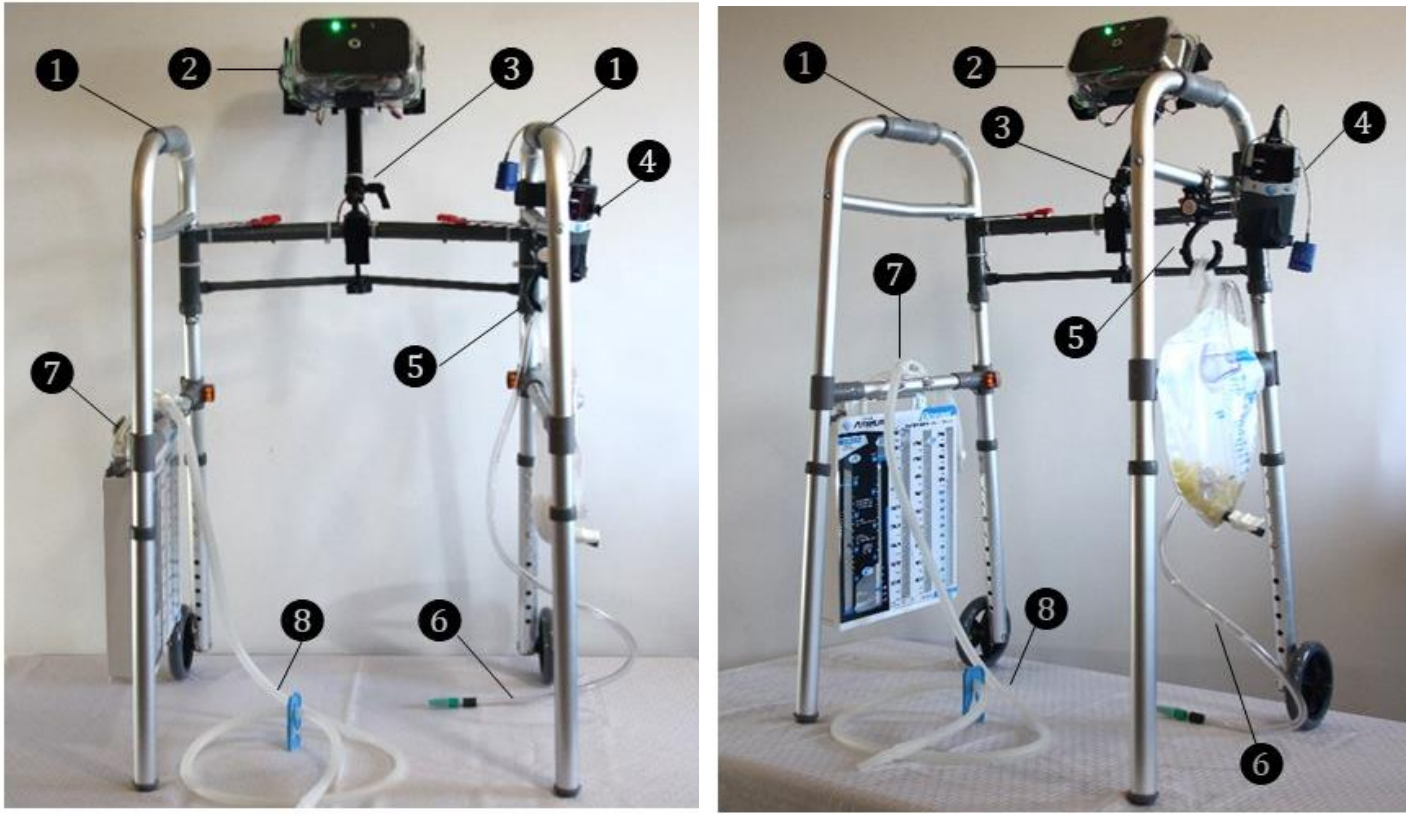

Figure 3: Clinical Force Measuring Walker v2.0 Components. 1) Integrated force transducers, 2) Force output electronic components, 3) Mounting bracket, 4) Oximeter attachment, 5) Urinary collection bag hook, 6) Foley Catheter, 7) Chest tube reservoir attachment, 8) Chest tube.

The swivel hook was initially mounted using a Velcro strap, and attaching it with a hose clamp removed a porous component that would be difficult to disinfect.

A bracket fabricated to hold a standard handheld oximeter and was clamped to the right upper vertical walker support. This position allowed healthcare providers to maintain line-of-sight for continuous oxygen saturation and heart rate monitoring. In this position, the oximeter probe remained attached to the patient's finger while using the walker. The oximeter holder could also be placed on the left side or other horizontal or vertical walker support. The bracket was designed to make the digital display visible and allow easy oximeter placement and access to the power switch (Figure 4.D). A swivel hook was mounted to the upper right horizontal support using a metal hose clamp to suspend a urinary collection bag (Figure 4.B). A 90 degree swivel hook was used so urinary collection bags with a parallel or perpendicular suspension hook could be attached to the walker and maintain bag orientation in the same plane as the right walker support frame. By moving this swivel hook to a higher location, the S-hook to tether the Foley Catheter was no longer needed. This higher attachment location ensured that the urinary collection bag would not drag on the floor; it also could not swing inward, hitting the patient's foot because the lower right horizontal support blocked this motion. Even with this higher location, the urinary collection bag was still lower than the level of the bladder to maintain gravity assist drainage. The swivel hook was initially mounted using a Velcro strap, and attaching it with a hose clamp removed a porous component that would be difficult to disinfect.

A bracket to suspend the chest tube reservoir from the outside of the lower left horizontal walker support was fabricated (Figure 4.F). The bracket was $7.7 \mathrm{~cm}$ wide to keep the chest tube reservoir parallel to the walker side support and prevent front-toback movement, which is important to preserve the water seal required to maintain negative pleural pressure and lung inflation. The bracket was $5.1 \mathrm{~cm}$ deep with a wedge-shaped spacer, so the chest tube reservoir handle would slide into the bracket and then be cradled tightly to prevent side-to-side movement. The CFM
Walker v1.0 had a bracket mounted below the left lower horizontal support, which positioned the chest tube reservoir very close to the floor and front walker wheel. The new position not only moved the reservoir higher but to the outside rather than below it so any sideto-side movement would no longer interfere with a patient's gait.

\begin{tabular}{|c|c|c|c|}
\hline \multicolumn{2}{|c|}{ RIGHT } & \multicolumn{2}{|c|}{ LEFT } \\
\hline Force & LED & Force & LED \\
\hline 1.0 & G & 20 & G \\
\hline 1.2 & G & 2.2 & G \\
\hline 1.6 & G & 2.6 & G \\
\hline 2.0 & G & 3.2 & G \\
\hline 22 & G & 4.0 & G \\
\hline 24 & G & 4.4 & G \\
\hline 3.0 & G & 4.6 & G \\
\hline 3.4 & G & 5.0 & G \\
\hline 3.6 & G & 5.0 & G \\
\hline 4.2 & G & 5.6 & G \\
\hline 4.4 & G & 6.2 & G \\
\hline 4.8 & G & 7.0 & G \\
\hline 5.0 & G & 7.2 & G \\
\hline 5.8 & G & 76 & $Y$ \\
\hline 5.8 & G & 7.8 & $Y$ \\
\hline 6.2 & G & 8.4 & $Y$ \\
\hline 6.4 & G & 8.4 & $Y$ \\
\hline 7.0 & $\mathbf{Y}$ & 8.6 & $Y$ \\
\hline 8.0 & $Y$ & 8.8 & $Y$ \\
\hline 9.2 & $Y$ & 9.2 & $Y$ \\
\hline 9.2 & $Y$ & 9.6 & $\mathbf{R}$ \\
\hline 9.8 & $\mathbf{R}$ & 9.8 & $\mathbf{R}$ \\
\hline 10.0 & $\mathbf{Y}$ & 10.4 & R \\
\hline 10.6 & $\mathbf{R}$ & 10.8 & R \\
\hline 11.6 & $\mathbf{R}$ & 12.4 & $\mathrm{R}$ \\
\hline 11.8 & $\mathbf{R}$ & 14.0 & $\mathrm{R}$ \\
\hline 13.2 & R & 15.6 & R \\
\hline 15.4 & $\mathbf{R}$ & 16.2 & $\mathbf{R}$ \\
\hline 17.4 & $\mathbf{R}$ & 176 & $\mathrm{R}$ \\
\hline 23.8 & $R$ & 20.0 & R \\
\hline Error\% & $10.3 \%$ & & $10.3 \%$ \\
\hline
\end{tabular}

Figure 5: Force (lb) accuracy testing results. $\mathbf{G}=$ green, $\mathbf{Y}=$ yellow, $\mathbf{R}=$ red, Bold $=$ Error 


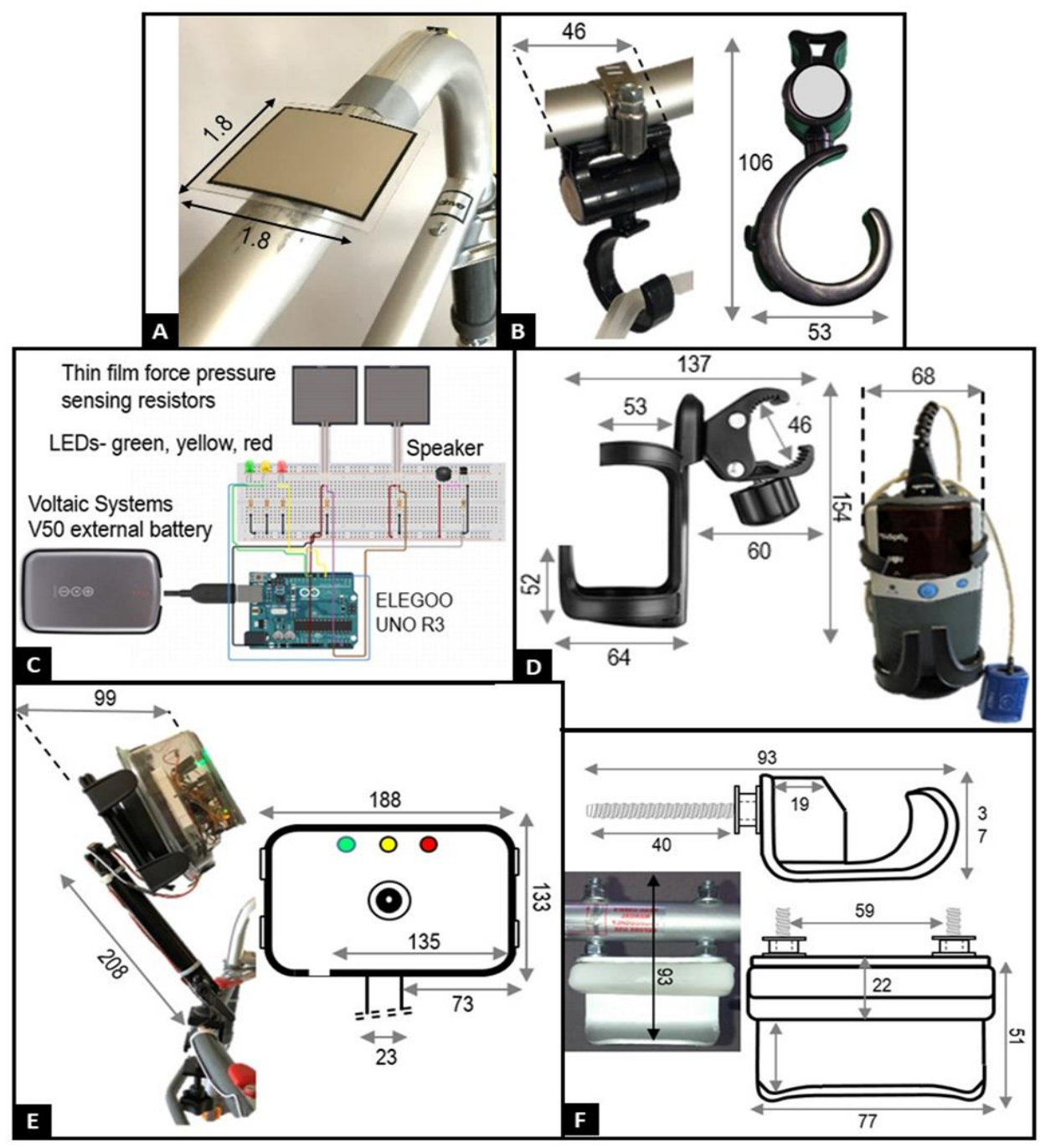

Figure 4: Components of the Clinical Force Measuring Walker v2.0. A) Thin film force resistors, B) Urinary collection bag hook, C) Arduino circuit components, E) Feedback console components, D) Oximeter holder, and F) Chest tube reservoir bracket.

All measurements in mm.

Lastly, the walker's legs were modified slightly based on the qualitative data and engineering testing. The color-coding was removed because the rehabilitation professionals said it was not necessary (they are used to changing walker heights), and the tape was somewhat porous, so there were concerns about adequately disinfecting it. The study participants overwhelmingly preferred $12.7 \mathrm{~cm}$ single-plane wheels on the front with regular legs on the back of the walker. They stated that this configuration is what they use in clinical settings and prescribe for patients' home assistive devices. Walker drag was tested (Table 3 ) using 4 types of front wheels (12.7 cm single-plane, $12.7 \mathrm{~cm}$ swivel, $7.6 \mathrm{~cm}$ single-plane, and $7.6 \mathrm{~cm}$ swivel). Results showed that with $12.7 \mathrm{~cm}$ single-plane front wheels walker horizontal push-pull resistance was significantly less than with the other wheels on smooth, solid surface flooring (similar to that found in a hospital) both with and without medical equipment attached. Three types of "ski-like" gliders on the back walker legs were tested with the $12.7 \mathrm{~cm}$ single-plane wheels, and results suggested that none significantly reduced walker drag and in many cases increased it.

The total cost for the components to fabricate the CFM Walker v2.0 was $\$ 238$. The total cost did not include the actual medical devices (portable oxygen tank, chest tube, chest tube reservoir, urinary collection bag, and Foley / urinary catheter). By removing the externally mounted force transducers and creating a simple force feedback interface using an Arduino system, the cost ( $\$ 1,171$ savings) and weight (4.5 $\mathrm{kg}$ reduction) of the CFM Walker v2.0 were substantially reduced. The CFM Walker v2.0 met all criteria/constraints, and the testing results are shown in Table 4. In addition, technical diagrams of the CFM Walker v2.0 are shown in Figure 6.

\section{Discussion}

The interview data obtained supported the overall clinical need for an assistive device with the ability to provide feedback regarding UEWB. This information was used to guide revisions of the CFM Walker v1.0 and engineering of the CFM Walker v2.0. Several specific alterations were made to the second walker prototype based on the qualitative data. Getting rid of the externally mounted force transducers and finding a force measuring mechanism that would be integrated and streamlined was a top priority. It was also necessary to design a force output display that was much simpler for patients to interpret and that had upper limit visual and auditory signals. Revisions to the medical equipment included removing the oxygen tank bracket and Foley Catheter S-hook. The only addition suggested was an attachment to hold a pulse oximeter. For the device to be easily disinfected, all components needed to be nonporous and water-resistant, so replacing some materials was needed.

Finally, follow-up qualitative interviews were conducted with the rehabilitation professionals. Study participants were able to use the CFM Walker v2.0 and test the force measuring system 
and medical equipment attachments. They were provided a brief overview of the CFM Walker v1.0 revisions incorporated into the CFM Walker v2.0. Then they were asked these general questions - What is your opinion of the: 1) force transducers integrate into the walker handles? 2) force feedback display and buzzer? 3) electronic components housing and mounting arm? 4) oximeter holder? 5) urinary collection bag swivel hook? 6) chest tube reservoir bracket? and 7) overall walker performance?

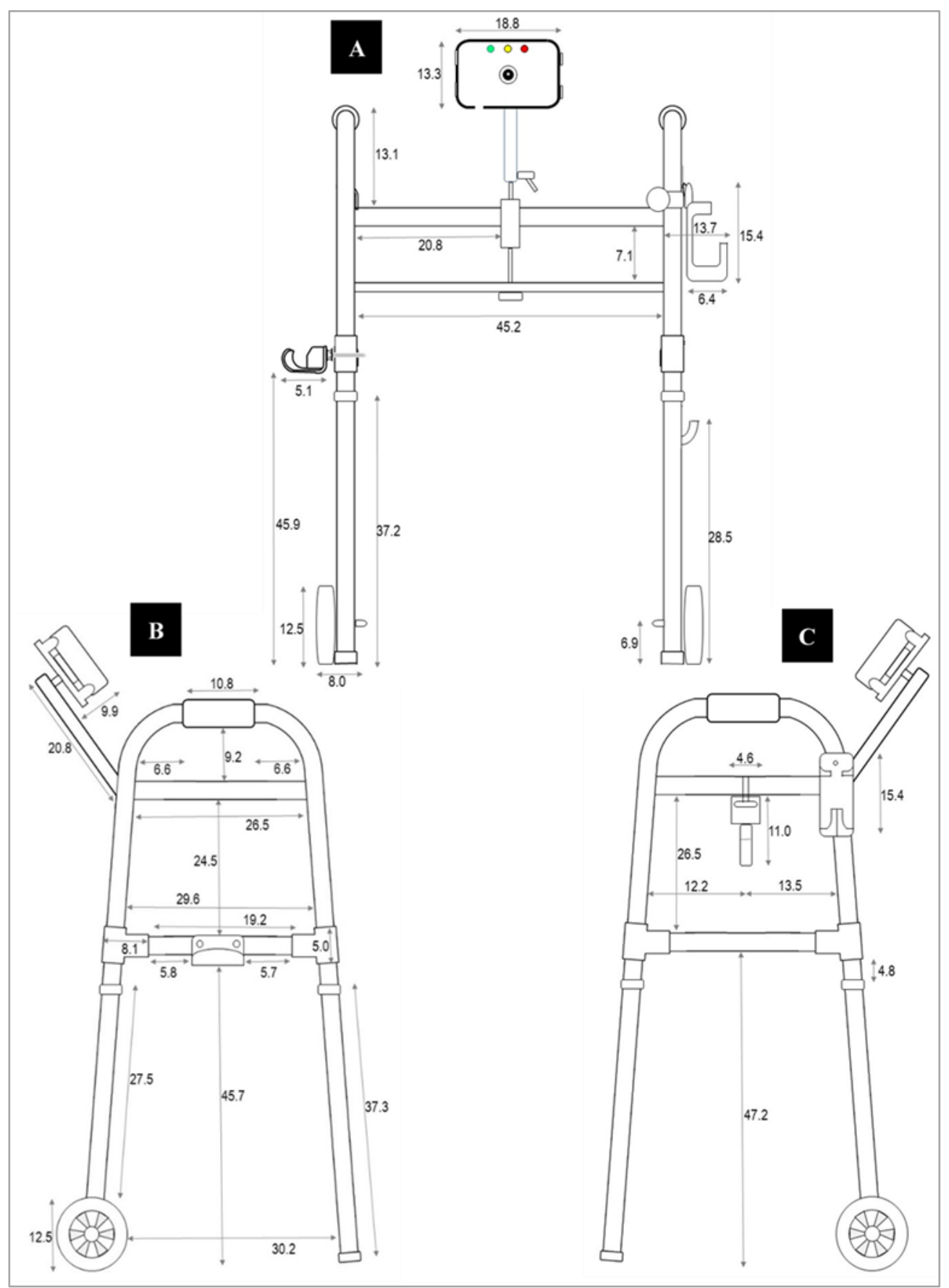

Figure 6: Schematic diagrams of the Clinical Force Measuring Walker v2.0. A) Back view, B) Left side view, and C) Right side view. All measurements in cm

Table 3: Horizontal Push-Pull Resistance (kg) for 4 Different Front Wheel Types. $P<0.05$

\begin{tabular}{|l|l|l|l|l|l|l|l|l|}
\hline & $\mathbf{1 2 . 7}$ Planar Wheels & \multicolumn{2}{l|}{$\mathbf{1 2 . 7} \mathbf{~ c m}$ Swivel Wheels } & \multicolumn{2}{l|}{$\mathbf{7 . 6}$ cm Planar Wheels } & \multicolumn{2}{l|}{ 7.6 cm Swivel Wheels } \\
\hline Trial \# & Push & Pull & Push & Pull & Push & Pull & Push & Pull \\
\hline 1 & 0.7 & 1.3 & 0.6 & 1.0 & 0.8 & 2.4 & 0.9 & 2.1 \\
\hline 2 & 0.7 & 0.8 & 0.8 & 1.6 & 0.7 & 1.8 & 1.0 & 1.8 \\
\hline 3 & 0.8 & 1.0 & 0.6 & 1.4 & 0.9 & 2.4 & 0.9 & 2.6 \\
\hline 4 & 0.7 & 0.9 & 0.6 & 1.7 & 0.8 & 1.7 & 1.1 & 2.7 \\
\hline 5 & 0.8 & 1.1 & 0.9 & 1.6 & 0.8 & 1.8 & 1.1 & 2.5 \\
\hline 6 & 0.8 & 1.4 & 0.7 & 1.4 & 0.8 & 2.2 & 0.9 & 2.2 \\
\hline 7 & 0.8 & 1.5 & 0.8 & 1.2 & 0.8 & 2.3 & 0.8 & 2.2 \\
\hline 8 & 0.8 & 1.1 & 0.7 & 1.8 & 0.8 & 2.5 & 1.1 & 2.2 \\
\hline
\end{tabular}




\begin{tabular}{|l|l|l|l|l|l|l|l|l|}
\hline 9 & 0.7 & 1.2 & 0.9 & 1.2 & 0.8 & 2.0 & 0.9 & 2.3 \\
\hline 10 & 0.8 & 1.2 & 0.8 & 1.4 & 0.8 & 2.0 & 1.1 & 2.1 \\
\hline Mean & 0.76 & 1.15 & 0.74 & $1.43^{*}$ & 0.80 & $2.11^{* \dagger}$ & 0.98 & $2.27^{* \dagger}$ \\
\hline SD & 0.05 & 0.22 & 0.12 & 0.25 & 0.05 & 0.29 & 0.11 & 0.27 \\
\hline
\end{tabular}

*Significantly greater than push; †Significantly greater than $12.7 \mathrm{~cm}$ Wheel

Table 4: Comparison of Walker Versions' Design Criteria and Constraint Characteristics.

\begin{tabular}{|c|c|c|c|}
\hline Design Elements & Research Device & CFM Walker v1.0 & CFM Walker v2.0 \\
\hline Vertical force measuring capability & Accuracy $=82 \%$ & Accuracy $=82 \%$ & Accuracy $=90 \%$ \\
\hline Ergonomic handles & Diameter $=2.2 \mathrm{~cm}$ & Diameter $=5.2 \mathrm{~cm}$ & Diameter $=3.5 \mathrm{~cm}$ \\
\hline $\begin{array}{l}\text { Simple visual \& auditory feedback } \\
\text { with alarms }\end{array}$ & $\begin{array}{l}\text { Visual } \rightarrow<50 \mathrm{~cm} \\
\text { Auditory } \rightarrow \text { NONE }\end{array}$ & $\begin{array}{l}\text { Visual } \rightarrow<50 \mathrm{~cm} \\
\text { Auditory } \rightarrow \text { NONE }\end{array}$ & $\begin{array}{l}\text { Visual display } \rightarrow>3 \mathrm{~m} \\
\text { Auditory signal } \rightarrow>3 \mathrm{~m}\end{array}$ \\
\hline $\begin{array}{l}\text { Streamlined, stable, \& maneuverable } \\
\text { frame }\end{array}$ & $\begin{array}{l}\text { Width }=67.9 \mathrm{~cm} \\
\text { Depth }=\mathbf{5 3 . 0} \mathbf{~ c m}\end{array}$ & $\begin{array}{l}\text { Width }=67.9 \mathrm{~cm} \\
\text { Depth }=\mathbf{5 3 . 0} \mathbf{~ c m}\end{array}$ & $\begin{array}{l}\text { Width }=63.0 \mathrm{~cm} \\
\text { Depth }=50.2 \mathrm{~cm}\end{array}$ \\
\hline Lightweight construction & No $\mathrm{MD}=7.2 \mathrm{~kg}$ & $\begin{array}{l}\text { No MD }=8.4 \mathrm{~kg} \\
\text { With } \mathrm{MD}=13.0 \mathrm{~kg}\end{array}$ & $\begin{array}{l}\text { No MD = 3.9 kg } \\
\text { With MD = 5.6 kg }\end{array}$ \\
\hline Minimal drag (Push-Pull) & No $\mathrm{MD}=1.4-2.0 \mathrm{~kg}$ & $\begin{array}{l}\text { No } M D=1.4-2.0 \mathrm{~kg} \\
\text { With } \mathrm{MD}=1.4-2.0 \mathrm{~kg}\end{array}$ & $\begin{array}{l}\text { No MD = 0.5 - } 0.9 \mathrm{~kg} \\
\text { With } M D=0.8-1.2 \mathrm{~kg}\end{array}$ \\
\hline Adjustable height handles & $1.76-2.06 \mathrm{~m}$ & $1.76-2.06 \mathrm{~m}$ & $1.49-1.95 \mathrm{~m}$ \\
\hline Ability to disinfect & $\begin{array}{l}\text { Nonporous } \rightarrow \text { Yes } \\
\text { Water resistant } \rightarrow \text { Yes }\end{array}$ & $\begin{array}{l}\text { Nonporous } \rightarrow \text { No } \\
\text { Water resistant } \rightarrow \text { Yes }\end{array}$ & $\begin{array}{l}\text { Nonporous } \rightarrow \text { Yes } \\
\text { Water resistant } \rightarrow \text { Yes }\end{array}$ \\
\hline Affordable cost & $\$ 1,359$ & $\$ 1,409$ & $\$ 238$ \\
\hline
\end{tabular}

Bold indicates the testing criteria set was met; $M D=$ Medical Devices

\section{Conclusions}

In conclusion, previous research suggests that patients are not good at estimating UEWB force less than $4.5 \mathrm{~kg}$ and that feedback training is effective at reducing it ${ }^{[12-14,18,20,31]}$. Therefore using an instrumented walker and feedback training would be beneficial in clinical practice, especially with older patients. The qualitative data obtained from rehabilitation professionals (Part 1) indicated that a CFM walker with integrated handles would be clinically useful. Suggestions for the CFM Walker v1.0 led to modifications including, streamlining, altering, removing, and adding components. Finally, engineering tests of the CFM Walker v2.0 demonstrated that it met essential criteria for making it feasible for patients who need to limit UEWB to prevent excessive bone stress during post-fracture ossification. Ultimately the CFM Walker v2.0 could improve outcomes for patients recovering from heart surgery performed via median sternotomy and certain orthopaedic conditions that are associated with upper body bone fracture (iatrogenic or traumatic) and subsequent osteogenesis.

\section{Ethics approval and consent to participate}

This study was approved by the Eastern Washington University Institutional Review Board for Human Subjects (Protocol \#HS5953), and all subjects signed an informed consent prior to participation; procedures were in accordance with the Helsinki Declaration.

\section{List of abbreviations $\mathrm{CFM}=$ Clinical Force Measuring}

$\mathrm{G}=$ Green

LEDs $=$ Light Emitting Diodes

$\mathrm{MD}=$ Medical Devices

$\mathrm{R}=\mathrm{Red}$

UEWB = Upper Extremity Weight Bearing

v1.0 = First Prototype Version

v2.0 = Second Prototype Version

$\mathrm{Y}=$ Yellow

\section{Data Availability}

Transcripts and coding of the interview data can be obtained by request from the author.

\section{Conflicts of Interest}

The author declares that there is no conflict of interest regarding the publication of this paper.

\section{Funding Statement}

This research received no external funding.

\section{Authors' contributions}

AL was responsible for study design, data collection and analysis, device testing, interpretation of results, and writing the manuscript (all drafts and final versions).

\section{References}

[1] Tuyl LJ, Mackney JH, Johnston CL. Management of sternal precautions following median sternotomy by physical therapists in Australia: a web-based survey. Phys Ther 2012, 92(1), 83-97.

[2] Balachandran S, Lee A, Denehy L, et al. Risk factors for sternal complications after cardiac operations: a systematic review. Ann Thorac Surg 2016, 102(6), 21092117.

[3] Brocki BC, Thorup CB, Andreasen JJ. Precautions related to midline sternotomy in cardiac surgery: a review of mechanical stress factors leading to sternal complications. Eur J Cardiovasc Nurs 2010, 9(2), 77-84.

[4] Casha AR, Manche A, Gatt R, et al. Mechanism of median sternotomy dehiscence. Interact Cardiovasc Thorac Surg 2014, 19(4), 617-621. 
[5] Kostenuik P, Mirza FM. Fracture healing physiology and the quest for therapies for delayed healing and nonunion. J Orthop Res 35, 213-223, 2017

[6] Zubair MH, Smith JM. Updates in minimally invasive cardiac surgery for general surgeons. Surg Clin North Am 2017, 97(4), 889-898.

[7] Alhalawani AM, Towler MR. A review of sternal closure techniques. J Biomater Appl 2013, 28(4), 483-497.

[8] Min L, Mazzurco L, Gure TR, et al. Longitudinal functional recovery after geriatric cardiac surgery. J Surg Res 2015, 194(1), 25-33.

[9] Stocicea N, You T, Eiterman A, et al. Perspectives of post-acute transition of care for cardiac surgery patients. Front Cardiovasc Med 2017, 27(4), 70.

[10] Edgerton JR, Herbert MA, Mahoney C, et al. Long-term fate of patients discharged to extended care facilities after cardiovascular surgery. Ann Thorac Surg 2013, 96(3), 871-877.

[11] Graham A, Brown CH. Frailty, aging, and cardiovascular surgery. Anesth Analg 2011, 124(4), 1053-1060.

[12] LaPier A, Cleary K. Feedback training improves accuracy of estimating upper extremity weight bearing during functional tasks - implications after open heart surgery. Internat J Physiother Res 2019, 7(4), 3163-3172. DOI: 10.16965/ijpr.2019.151

[13] LaPier A, Cleary K. Feedback training improves compliance with sternal precaution guidelines during functional mobility: implications for optimizing recovery in older patients after median sternotomy. Appl Bionics Biomech 2021, Article ID 8889502:13 pages. doi.org/10.1155/2021/8889502

[14] LaPier A, Cleary K. The influence of age and feedback training on ability to modulate upper extremity weight bearing force and pectoralis major muscle recruitment while following sternal precautions. Phys Ther Rehabil 2021, 8, 1. dx.doi.org/10.7243/2055-2386-8-1

[15] Adams J, Cline MJ, Hubbard M, et al. A new paradigm for post-cardiac event resistance exercise guidelines. Am J Cardiol 2006, 97(2), 281-286.

[16] Swanson LB, Kinney LaPier T. Upper extremity forces generated during activities of daily living. J Acute Care Phys Ther 2014, 5(2), 70-76.

[17] Ruiz FK, Fu MC, Bohl DD, et al. Patient compliance with postoperative lower extremity touch down weight bearing orders at a level I trauma center. Orthop 2014, 37(6), e552-556.

[18] Hustedt JW, Blizzard DJ, Baumgaertner MR, Leslie MP, Grauer JN. Effect of age on partial weight-bearing training. Orthop 2012, 35(7), e1061-1067.

[19] Hustedt JW, Blizzard DJ, Baumgaertner MR, Leslie MP, Grauer JN. Is it possible to train patients to limit weight bearing on a lower extremity? Orthop 2012, 35(1), e3137.

[20] Hustedt JW, Blizzard DJ, Baumgaertner MR, Leslie MP, Grauer JN. Lower-extremity weight-bearing compliance is maintained over time after biofeedback training. Orthop 2012, 35(11), e1644-1648.

[21] Uhl TL, Carver TJ, Mattacola CG, Mair SD, Nitz AJ. Shoulder musculature activation during upper extremity weight-bearing exercise. J Orthop Sports Phys Ther 2003, 33(3), 109-117.

[22] Po-Chen Y, Cherng L. Using walker during walker: a pilot study for health elder. Work 2012, 41, 2081-2085.

[23] Costamagna E, Thies SB, Kenneyet LPJ, et al. A generalizable method for the assessment of static stability of walking aid users. Med Eng Physics 2017, 46, 167-175.

[24] Gill S, Scheme E. Design of a smart enabled walker or deployable activity and gait monitoring. IEEE Life Sci Conference, 2018. DOI:10.1109/LSC.2018.8572227

[25] Khodadadi M, Baniasad MA, Arazpour M, Farahmand F, Zohoor H. Designing instrumented walker to measure upper-extremity's efforts: a case study. Assist Tech 2018, 26, 1-9.

[26] Alwen M, Rajendran PJ, Ledoux A, Huang C, Wasson G, Shethet P. Stability margin monitoring in steeringcontrolled intelligent walkers for the elderly. AAAI Fall Symposium: Caring Machines, FS05-02-001, 2005. https://www.aaai.org/Papers/Symposia/Fall/2005/FS-0502/FS05-02-001.pdf

[27] Bonzino J, Peterson D. Medical Devices and Human Engineering. 4th Ed. CRC Press: Boca Raton, FL, USA, 2015.

[28] Paz J, West. Acute Care Handbook for Physical Therapists. Elsevier: St. Louis, MO, USA, 2014.

[29] Neergaard MA, Olesen F, Andersen RS, Sondergaard J. Qualitative description - The poor cousin of health research? BMC Med Res Methodology 2009, 9, 52.

[30] Creswell J. Qualitative Inquiry and Research Design. Sage: Thousand Oaks, CA, USA, 1998.

[31] Sawers A, Hahn ME, Kelly VE, Czerniecki JM, Kartin D. Beyond componentry: how principles of motor learning can enhance locomotor rehabilitation of individuals with lower limb loss - a review. J Rehabil Res Dev 2012, 49(10), 1431-1442.

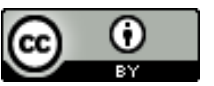

Open Access This article is licensed under a Creative Commons Attribution 4.0 International License, which permits use, sharing, adaptation, distribution and reproduction in any medium or format, as long as you give appropriate credit to the original author(s) and the source, provide a link to the Creative Commons license, and indicate if changes were made. The images or other third party material in this article are included in the article's Creative Commons license, unless indicated otherwise in a credit line to the material. If material is not included in the article's Creative Commons license and your intended use is not permitted by statutory regulation or exceeds the permitted use, you will need to obtain permission directly from the copyright holder. To view a copy of this license, visit https://creativecommons.org/licenses/by/4.0/.

(C) The Author(s) 2021 